ICU, 14\% [before patient contact] and 25\% [after patient contact]; cardiac surgery ICU, $6 \%$ [before] and 13\% [after]). Observations in the medical ICU after introduction of the new, increasingly accessible, alcohol-based, waterless hand antiseptic revealed significantly higher handwashing rates $(P<.05)$. Handwashing compliance improved as accessibility was enhanced: before patient contact, $19 \%$, and after contact, $41 \%$, with one dispenser per four beds; and before contact, $23 \%$, and after contact, $48 \%$, with one dispenser for each bed.

The authors concluded that the education and feedback intervention and the patient awareness program failed to improve handwashing compliance. However, introduction of easily accessible dispensers with an alcohol-based waterless handwashing antiseptic led to significantly higher handwashing rates among healthcare workers.

FROM: 1. Maury E, Alzieu M, Baudel JL, Haram N, Barbut F, Guidet B, et al. Availability of an alcohol solution can improve hand disinfection compliance in an intensive care unit. Am J Respir Crit Care Med 2000;162:324-327.

2. Bischoff WE, Reynolds TM, Sessler CN, Edmond $\mathrm{MB}$, Wenzel RP. Handwashing compliance by health care workers: the impact of introducing an accessible, alcoholbased hand antiseptic. Arch Intern Med 2000;160:1017-1021.

\section{Heat-Moisture Exchangers and Risk of Nosocomial Pneumonia}

Davis and colleagues, at the University of Cincinnati, studied the effect of using a single heat-and-moisture exchanger (HME) for $\leqslant 120$ hours on the efficiency, resistance, level of bacterial colonization, frequency rate of nosocomial pneumonia, and cost compared with changing the HME every 24 hours. In a prospective, randomized, controlled study in a surgical ICU, the study population included 220 consecutive patients requiring mechanical ventilation for 48 hours. Patients were randomized to one of three groups: (1) hygroscopic HME (Aqua+) changed every 24 hours (HHME24); (2) hydrophobic HME (duration HME) changed every 120 hours (HME-120); and (3) hygroscopic HME (Aqua+) changed every 120 hours (HHME-120). Devices in all groups could be changed at the discretion of the staff when signs of occlusion or increased resistance were identified.

Daily measurements of inspired gas temperature, inspired relative humidity, and device resistance were made. Additionally, daily cultures of the patient side of the device were accomplished. The frequency rate of nosocomial pneumonia was made by using clinical criteria. Ventilatory support variables, airway care, device costs, and clinical indicators of humidification efficiency (sputum volume, sputum efficiency) also were recorded.

Prolonged use of both hygroscopic and hydrophobic devices did not diminish efficiency or increase resistance. There was no difference in the number of colony-forming units (CFUs) from device cultures over the 5-day period and no difference between CFUs in devices changed every 24 hours compared with devices changed after 120 hours. The average duration of use was $23 \pm 4$ hours in the HHME-24 group, $73 \pm 13$ hours in the HME-120 group, and $74 \pm 9$ hours in the HHME-120 group. Mean absolute humidity was greater for the hygroscopic devices $\left(30.4 \pm 1.1 \mathrm{mg}\right.$ of $\left.\mathrm{H}_{2} \mathrm{O} / \mathrm{L}\right) \mathrm{com}$ pared with the hydrophobic devices $\left(27.8 \pm 1.3 \mathrm{mg}\right.$ of $\left.\mathrm{H}_{2} \mathrm{O} / \mathrm{L}\right)$.

The frequency rate of nosocomial pneumonia was $8 \%$ (8:100) in the HHME-24 group, 8.3\% (5:60) in the HME-120 group, and 6.6\% (4:60) in the HHME-120 group. Pneumonia rates per 1,000 ventilatory-support-days were $20: 1,000$ in the HHME-24 group, 20.8:1,000 in the HME-120 group, and $16.6: 1,000$ in the HHME-120 group. Costs per day were $\$ 3.24$ for the HHME-24 group, $\$ 2.98$ for the HME-120 group, and $\$ 1.65$ for the HHME-120 group.

The authors concluded that changing the hydrophobic or hygroscopic HME after 3 days does not diminish efficiency, increase resistance, alter bacterial colonization, or increase the rate of nosocomial pneumonia. Thus, use of HMEs for $>24$ hours, up to 72 hours, is safe and cost-effective.

FROM: Davis K Jr, Evans SL, Campbell RS, Johannigman JA, Luchette FA, Porembka DT, et al. Prolonged use of heat and moisture exchangers does not affect device efficiency or frequency rate of nosocomial pneumonia. Crit Care Med 2000;28:1412-1418.

\section{Semiquantitative Culture of IV Catheter Without Removal}

Sensitivity and negative predictive values of combined surface cultures (skin and hub) are high in the presumptive diagnosis of catheter-related infection, but specificity and positive predictive values (PPVs) are poor. Fortun and coinvestigators from Madrid, Spain, conducted a prospective study to evaluate the yield of the semiquantitative culture of the subcutaneous segment in the diagnosis of colonization of the catheter tip without removal of the catheter.

One hundred twenty-four nontunneled central venous catheters were removed because of suspected infection or the end of therapy. Colonization was considered if $>15$ colonyforming units (CFUs) in the roll procedure or $>1,000$ CFUs in the quantitative Cleri procedure were recovered from the tip cultures (gold standard). Before removing the catheter, a semiquantitative culture of skin surrounding the point of insertion, a semiquantitative culture of the subcutaneous segment (after removing the catheter only $2 \mathrm{~cm}$ ), a semiquantitative culture of the hub, and a quantitative blood culture were performed. Receiver operating characteristic curves were calculated to estimate the cutoff points. A culture was considered positive when CFUs were $\geqslant 15, \geqslant 15$, and $\geqslant 5$ for skin, hub, and subcutaneous segment cultures, respectively.

Colonization was detected in 51 catheters. The mean duration of catheterization was $14 \pm 8$ days; the rates of incidence of tip colonization and bacteremia were 2.9 per 100 catheter days and 1.2 per 100 catheter days, respectively. Sensitivity of skin, subcutaneous, and hub cultures analyzed individually were $\leqslant 61 \%$; however, specificity and PPVs of subcutaneous segment cultures were significantly higher than skin cultures (94\% and $88.5 \%$ vs $71.6 \%[P=.001]$ and $62 \%$ [ $P=.014]$, respectively). Sensitivity of the combined skin and hub cultures and of the combined subcutaneous segment and hub cultures were similar: $86.2 \%$ and $84.3 \%$, respectively; how- 
ever, specificity and PPVs of the latter combination were significantly higher than the former: $82 \%$ and $78.1 \%$ versus $59.7 \%$ $(P=.008)$ and $61.9 \%(P=.07)$, respectively. The likelihood ratio of a positive test for the combined subcutaneous segment and hub culture was 4.68 , and only 2.13 for the combined skin and hub culture.

The results indicate that the combined subcutaneous segment and hub culture constitute an easy, effective procedure for the conservative diagnosis of catheter colonization.

FROM: Fortun J, Perez-Molina JA, Asensio A, Calderon C, Casado JL, Mir N, et al. Semiquantitative culture of subcutaneous segment for conservative diagnosis of intravascular catheter-related infection. J Parenter Enteral Nutr 2000; 24:210-214.

\section{Bacteremia After Transrectal Ultrasound- Guided Prostate Biopsy}

Lindbert and coinvestigators, from the Department of Urology, Stanford University Medical Center, determined the incidence and predisposing factors of bacteremia and bacteriuria after prostate biopsy with specific emphasis on the value of a pre-biopsy enema. They randomized 50 men undergoing ultrasound and biopsy to rule out prostate cancer to receive a preoperative enema ( 25 men) or no enema ( 25 men). Preoperatively, urine was obtained for culture, and questionnaires regarding urological history and voiding symptoms were completed. Cultures were taken from the initial prostate biopsy, biopsy needle, and postoperative urine and blood specimens. The following day, a symptom questionnaire was completed.

Bacterial growth in post-procedure cultures did not correlate with the number of biopsies, prostate-specific antigen, obstructive voiding symptoms, prostate volume, cancer, or post-biopsy hematuria. Bacteriuria was noted in $44 \%$ of the cases, and bacteremia was present in $16 \%$ of the patients, of whom $87.5 \%$ did not receive an enema $(P=.0003)$. One patient had chills and fever $>37.5^{\circ} \mathrm{C}$, requiring additional antibiotics. On the follow-up questionnaire, $12 \%$ of patients described dysuria, including $84 \%$ with bacteriuria after biopsy.

The authors concluded that bacteremia and bacteriuria are common, but usually asymptomatic, after multiple biopsies. Bacteria apparently are introduced into the urine or blood from the rectum via the biopsy needle, which may be minimized by a pre-biopsy enema. Dysuria or a history of urinary tract infection did not predict problems after biopsy.

FROM: Lindert KA, Kabalin JN, Terris MK. Bacteremia and bacteriuria after transrectal ultrasound guided prostate biopsy. J Urol 2000;164:76-80.

\section{Healthcare Worker-to-Patient Transmission of $\mathrm{HCV}$ in the UK}

Several recent look-back investigations in the United Kingdom have demonstrated transmission of hepatitis $\mathrm{C}$ virus $(\mathrm{HCV})$ from infected healthcare workers $(\mathrm{HCWs})$ to patients during exposure-prone procedures. Since October
1999, investigations involving three $\mathrm{HCV}$-infected $\mathrm{HCW}$ s and testing of more than 6,000 patients have identified 12 patients who likely contracted HCV from their healthcare providers, according to the UK Department of Health. The three HCWs were unaware they were infected with $\mathrm{HCV}$ until 1 of their patients presented with infection. In each instance, subsequent testing confirmed $\mathrm{HCV}$ infection in the $\mathrm{HCW}$ and led to a more extensive look-back investigation.

The Department of Health and the hospitals involved would not provide information on the specific occupations of the workers or what procedures led to the spread of infection. $\mathrm{ABBC}$ news report, however, identified one as a surgeon in obstetrics and gynecology. The BBC speculated that another $\mathrm{HCW}$ also is a surgeon.

$\mathrm{HCV}$ infection was identified by the presence of hepatitis C RNA in blood samples. Samples positive for hepatitis C RNA were then genotyped and underwent hepatitis C RNA sequence analysis. Samples from patients and HCWs were then compared.

An investigation associated with an HCW in Boston, Lincolnshire, involved contacting and testing 4,500 patients treated by the worker at 11 hospitals in England and Wales after 1978. The UK National Health Service (NHS) organized an initial investigation in October 1999 after it was discovered that an $\mathrm{HCW}$ at Pilgrim Hospital in Boston transmitted $\mathrm{HCV}$ to a patient. NHS patient records were searched at hospitals where the HCW was based after 1993. In March, the UK Advisory Panel for Health Care Workers Infected with Bloodborne Viruses advised extending the investigation to 1978. So far, 8 patients appear to have contracted HCV from the worker.

A second investigation of a London-based $\mathrm{HCW}$ involved contacting approximately 1,900 patients in March who were treated between 1994 and 1999 at three London hospitals. Data collected to date have identified 3 patients who likely contracted infection from the $\mathrm{HCW}$.

A third investigation, which started on June 2, involves an HCW in Birmingham. Of 749 patients contacted, 1 patient has been found to be infected with $\mathrm{HCV}$ thus far.

Infected HCWs in the United Kingdom currently are advised not to perform exposure-prone procedures. Currently, no recommendations exist in the United States or elsewhere to restrict professional activities of $\mathrm{HCWs}$ with $\mathrm{HCV}$ infection. The $\mathrm{CDC}$ recommends that $\mathrm{HCV}$-positive HCWs, follow strict aseptic technique and Standard Precautions, including appropriate use of hand washing, protective barriers, and care in the use and disposal of sharp instruments.

In addition to the cases identified in this report, two incidents of transmission have been reported, one in Spain and one in London. In both, HCV transmission occurred during cardiothoracic surgery.

FROM: Adapted from news story in icanPrevent News (www.ican.prevent.com). Original source of information: UK Department of Health, June 7 press release: "Hepatitis C infected healthcare workers" and UK Department of Health, March 30 press release, with list of hospitals involved in the investigation: " 4,500 patients notified of potential exposure to infection." 